\title{
Calculating the orbits of comets
}

\section{The return of Halley's comet may have been a disappointment, but new-found skill at calculating cometary orbits may help towards an understanding of these objects.}

THE covert return of Halley's comet has been a great public frustration that may yet send hordes of people scurrying off to the South Pacific in March and April, after the perihelion passage predicted for 9 February 1986, just six weeks from now. In reality, the likelihood that very few people would ever see the comet was well signalled in advance; the Earth is on the wrong side of its orbit to make Halley the spectacular object it seems to have been in 1066 and 1682 . At a perihelion distance of 0.59 astronomical units, there can be a tenfold variation in the apparent brightness of the comet with the position of the Earth. At this unfavourable passage, many people will have to be content with reading about the event, in which case they might do worse than browse through the proceedings of last year's International Astronomical Union symposium on the dynamics of comets, which have now been published*.

Comets have an obviously special place in planetary dynamics as naturally occurring test particles in attempts at the solution of the many-body problem in celestial mechanics. Unlike the other objects in the Solar System whose mass is negligible compared with that of planets, they have the particular advantage of moving in orbits that are often far from being circular. Given the interest in spacecraft orbits and the availability of computer power, not to mention the inherent interest of the subject (and even of Halley's comet), it is inevitable that people should have been so busily calculating cometary orbits.

But is that not simply a matter of solving a few differential equations? There are two practical snags. Comets are not ideal test particles, but self-accelerating objects as a consequence of the way in which they lose mass asymmetrically near perihelion. And even the best-known, among which Halley and Encke (found at the end of the eighteenth century) stand out, travel in orbits whose elements are poorly known, at least in comparison with the orbits of the planets.

Running through last year's symposium seems to have been a hymn of gratitude to B.G. Marsden from the HarvardSmithsonian Institute of Astrophysics for his compilation over many years of an authoritative list of the orbital elements of more than a hundred periodic comets and for having, during the past two decades, founded the small but flourishing school of those who would put the treatment of the "non-gravitational" influences on cometary orbits on a rational basis.

What began as an empirical attempt to calculate the acceleration (sometimes the deceleration) of the comet from the changes in its orbit, so as to learn something of the physics of the processes responsible, has been rationalized by supposing extra forces which are functions of the heliocentric distance. On this view, comets accelerate by the transfer of momentum from the escaping gases; for the case of Encke, Y.V. Batrakov and Y.A. Chernetenko from the Institute of Theoretical Astronomy at Leningrad now think that the non-gravitational forces can be related to the magnetic index of solar activity, which makes sense.

In practice, the outcome of these calculations seems as satisfactory as could be expected. The assumption that the nongravitational force on a comet near perihelion is simply a function of the heliocentric distance and, specifically, that it is a power law works well enough. But the idea that the force could be in any direction than that towards the Sun requires that the nucleus of the comet should be spinning, whence the hope that the Giotto encounter next March may directly throw light on the revolution of the Halley nucleus.

Even without these qualifications, the solution of the differential equations of the motion of a comet is far from being simple. One obvious difficulty is that even the comets with very short periods are known accurately only from their brief passage through perihelion, so that integration entails projecting a set of nonlinear equations forward for the equivalent of many years from a poorly known starting point. So far as can be told from last year's meeting, computers have made possible several new ways of tackling the problem, which is why it is (or should be) all the more humbling that, at the very beginning of the eighteenth century, Halley had applied methods to the calculation of twenty cometary orbits and that, by the middle of the century, A.C. Clairaut had been able to allow for the effects of Saturn and Jupiter on the orbit of Halley's comet so as to predict its passage in 1757 to within a month.

The neatest, or at least the most ambitious of all the programmes of calculation described last year, is that which yields comet orbits as by-products of a continuing international project to calculate the evolution of the Solar System as a whole, known as the Long-Term Evolution Project. The project has the virtue of involving not merely people from Western Europe (among whom Italians are prominent), but also at least one group from Czechoslovakia. The objective is that the motion of the principal objects of the Solar System should be calculated almost from first principles. As with other numerical schemes of this kind, among which that of Edgar Everhart from the University of Denver is the best known, the proof of the pudding consists not of the agreement between predictions and future observations, but of agreement between the calculations and past records of the passage of known comets - "postdiction" as it is anachronistically called.

Many of the results really are quite startling, familar though they may be to those working in the field. Even Halley is not as well-behaved as might be thought. D.K. Yeomans of the Jet Propulsion Laboratory describes the attempts made since Halley's own work in 1705 , including that of Bessel in the early nineteenth century and of those this century who have had the benefit of the ancient Chinese records, only to conclude that this long series of perihelia, if used as the basis of strictly dynamical interpolation, foreshortens each cycle of roughly 76 years by about four days. With the discrepancy explained by the transfer of momentum at evaporation of the surface material, Yeomans concludes that the orbit of Halley's comet can have changed only slowly during the past 18,000 years or so, but that there may have been some more profound interaction with Jupiter at some point.

This fits in well with the calculations made of the behaviour of other comets in the past. There is for example, the case of the comet Oterma, whose orbit lies between those of Jupiter and Saturn, which encountered Saturn in the late eighteenth century, which has just spent two decades as a distant satellite of Jupiter, but which is now likely to return to much what its former orbit was. This is one of many examples quoted at the symposium to illustrate how comets filter their way from the supposed Oort cloud of comets-in-waiting, at some distance beyond Neptune, by successive encounters with planets into the inner Solar System. The comets we see are the exceptions.

John Maddox

Dynamics of Comets: Thet Origin and Evolution (eds Carusi A. \& Valsecchi, G.B.) (Reidel, Dordrecht, 1985). 